Marília Jesus Batista ${ }^{1}$

Herenia Procopio Lawrence ${ }^{2}$

Maria da Luz Rosário de Sousa ${ }^{1}$
${ }^{1}$ Faculdade de Odontologia de Piracicaba, Universidade Estadual de Campinas. Av. Limeira 901, Areião. 13414-018 Piracicaba SP Brasil.mariliajbatista@ yahoo.com.br

${ }^{2}$ Departamento de Saúde Pública Bucal, Faculdade de Odontologia, Universidade de Toronto.

\title{
Classificação das perdas dentárias: fatores associados a uma nova medida em uma população de adultos
}

\author{
Tooth loss classification: factors associated \\ with a new classification in an adult population group
}

\begin{abstract}
This study evaluated tooth loss and factors associated with a new classification, which considers not only the number of teeth lost, but also the number and position that they occupied in the mouth. In Piracicaba, State of São Paulo, Brazil, 248 adults (20-64 year-olds) were examined using a household probability sample. The oral examinations followed the WHO criteria for caries and periodontal disease. Socioeconom$i c$, demographic and dental service use data were collected. The tooth loss outcome, based on tooth position and number of missing teeth, was analyzed by hierarchical multinomial logistic regression using a conceptual model. The mean number of missing teeth was 8.52 (DP = 9.24). For those who had lost up to 12 posterior teeth, age $(P R=$ $1.1)$ and low social class $(P R=2.6)$ were significant; for those who lost up to 12 including anterior teeth, age $(P R=1.1)$ and clinical attachment loss $>4 \mathrm{~mm}(P R=2.9)$; and for tooth loss in excess of 13 teeth, age $(P R=1.3)$, low social class $(P R=$ 3.8), and visiting a dentist due to emergency ( $P R$ =9.4) were significant. Age was associated with tooth loss. The classification made it possible to differentiate variables in accordance with position or the number of teeth lost.
\end{abstract}

Key words Adults, Regression analysis, Epidemiological surveys, Tooth loss, Oral health
Resumo O objetivo deste estudo foi avaliar a perda dentária e os fatores associados com uma nova classificação, que considera não apenas o número de dentes perdidos mas a posição que os mesmos ocupavam na boca. Foram examinados por amostragem probabilística 248 adultos (20 - 64 anos), de Piracicaba-SP, Brasil, segundo a cárie e a doença periodontal, critérios da OMS. Dados socioeconômicos, demográficos e de uso de serviço odontológico foram obtidos por questionário. O desfecho perdas dentárias, considerando a posição e o número de dentes perdidos, foi analisado por regressão logística multinomial hierárquica, utilizando modelo conceitual. A média de dentes perdidos foi $8,52( \pm 9,24)$ e apenas $28,3 \%$ não perderam dentes devido a doenças bucais. Dos que haviam perdido até 12 dentes posteriores, idade $(R P=1,1) e$ classe social baixa $(R P=2,6)$ foram significativas: os que perderam até 12, incluindo anteriores, idade $(R P=1,1)$ e bolsa periodontal $>4 \mathrm{~mm}(R P=$ 2,9); para perda acima de 13, a idade $(R P=1,3)$, classe social baixa $(R P=3,8)$; e ida ao dentista por emergência $(P R=9,4)$. Idade foi associada a perdas dentárias no presente estudo. A classificação permitiu diferenciar variáveis de acordo com a posição ou o número de dentes perdidos.

Palavras-chave Adultos, Análises de regressão, Levantamentos epidemiológicos, Perdas dentárias, Saúde bucal. 


\section{Introdução}

As doenças bucais constituem um problema de saúde pública e as perdas dentárias são suas principais sequelas, ocasionadas tanto pela falta de acesso à assistência odontológica que não seja mutiladora, quanto pelo sobretratamento caracterizado pelo ciclo restaurador repetitivo ${ }^{1}$. A perda dentária severa está entre as cem doenças que mais agravam a saúde da população mundial, segundo classificação do Global Burden Disease $2010^{2}$. Esta condição causa impacto na qualidade de vida tanto em relação ao número de dentes perdidos quanto em relação à posição que ocupam na arcada dentária ${ }^{3,4}$.

No Brasil, observa-se que adultos e idosos apresentam um alto percentual de dentes perdidos. No primeiro levantamento epidemiológico nacional em Saúde Bucal, em 1986, na região Sudeste os dentes perdidos corresponderam a $65,4 \%$ da experiência de cárie medida pelo índice CPOD (que é a soma dos dentes cariados, perdidos e obturados) em adultos de 35 a 44 anos, e no grupo de idosos (65 a 74 anos) $93 \%$ de dentes perdidos $^{5}$. Em 2010, quase 24 anos depois, os resultados apresentaram uma redução de 24,2 pontos percentuais, recuando a $41,2 \%$ na população adulta da mesma faixa etária ${ }^{6}$. Entretanto, a prevalência ainda é alta e manteve-se sem melhorias na população idosa ${ }^{6}$, sendo esta um importante foco de estudo.

A cárie e a doença periodontal são as condições clínicas que constituem os principais motivos da perda dentária em adultos ${ }^{7}$. Além das causas clínicas, estudos têm demonstrado que fatores demográficos, socioeconômicos, práticas relacionadas à saúde e utilização de serviço têm sido associados às perdas dentárias ${ }^{8-12}$.

É um fenômeno recente a utilização de estudos epidemiológicos populacionais como ferramenta para o planejamento de estratégias para melhoria da saúde bucal comparativamente a outras áreas da saúde ${ }^{13}$. O conhecimento dos fatores que desempenham um papel importante na saúde bucal e no desenvolvimento das doenças como o sistema de saúde oferecido e até o meio ambiente que o indivíduo está inserido, além de fatores como os individuais, familiares e da comunidade em que vivem, são importantes para determinar estratégias de promoção de saúde ${ }^{14}$, sendo imprescindível a abordagem populacional, que deve ser sempre considerada no cuidado odontológico e em saúde ${ }^{13}$.

A avaliação quantitativa e qualitativa da perda dentária, utilizando uma classificação que avalie numa mesma medida tanto a posição (qualitativa) quanto o número de dentes perdidos (quantitativa), faz-se necessária para o melhor entendimento de como se distribui a perda dentária na população. Tanto para o planejamento de reabilitação como na identificação dos fatores associados às diferentes condições de perdas dentárias. Diante do exposto, este estudo utilizou uma nova classificação, que considera a posição e o número dos dentes perdidos, para avaliar a perda dentária e os fatores associados a esta condição em uma população de adultos.

\section{Material e Métodos}

\section{Questões Éticas}

Em se tratando de um estudo com seres humanos, o mesmo foi submetido à avaliação e obteve aprovação pelo Comitê de Ética em Pesquisa da Faculdade de Odontologia de Piracicaba-Universidade Estadual de Campinas.

\section{Local do estudo}

Este estudo teve delineamento do tipo transversal e foi realizado no município de Piracicaba, localizado no Interior de São Paulo, Brasil. Entre junho e setembro de 2010, foi realizada a coleta de dados em domicílios da cidade que foram selecionados por amostragem probabilística.

\section{Amostra}

O cálculo amostral foi realizado com base nos dados obtidos no estudo de Batista et al. ${ }^{12}$, que examinou adultos de 20 a 64 anos. Considerando as diferenças existentes nas condições de saúde bucal nas diferentes faixas etárias, o tamanho amostral foi estimado para adultos de 20 a 44 anos e para adultos de 45 a 64 anos, utilizando dois cálculos. Adotou-se deff de 1,5, margem de erro de $10 \%$, e intervalo de confiança de $95 \%$, e dados de prevalência da experiência de cárie (soma de dentes cariados, perdidos e obturados) para cada grupo etário $(70,2 \%$ e $90,9 \%$ respectivamente), somando-se ao total de $20 \%$ para compensar eventuais perdas. $\mathrm{O}$ tamanho amostral para adultos de 20 a 44 foi 172 e, para adultos de 45 a 64 anos 68, estimando 240 indivíduos. Acrescentamos $30 \%$ ao tamanho amostral final, para a seleção dos domicílios, prevendo a possibilidade de perdas e recusas, resultando em 342 domicílios, sendo 11,4 domicílios por setor cen- 
sitário. Foram considerados para o sorteio 11 domicílios para cada um dos 30 setores censitários.

A seleção amostral foi planejada a partir de dados do censo do ano 2000 fornecidos pelo IBGE, que eram os dados compilados mais recentes no momento do estudo. A população de Piracicaba, em 2000, era 368.836 no total, sendo que 202.131 indivíduos se apresentavam entre 20 e $64 \operatorname{anos}^{15}$. A média de moradores na faixa etária por domicílio no censo de 2000 foi 2,4937 e a média de domicílios por setor foi $177,752^{15}$. Foram sorteados, segundo a técnica de amostragem probabilística, 30 setores censitários, mais 2 para serem suplentes, caso houvesse necessidade. Em cada setor foram sorteadas 11 residências, segundo a fração que variou em cada setor censitário e que foi determinada pelo número de domicílios. Foi examinado um adulto por domicílio.

\section{Variáveis}

Os exames clínicos bucais foram realizados nos domicílios, com iluminação natural (sem profilaxia prévia, nem secagem), utilizando sondas CPI e espelhos bucais planos como preconizados pela Organização Mundial da Saúde (OMS, 1987). O único examinador foi treinado com discussões teóricas e práticas, e calibrado por total de dezesseis horas obtendo no mínimo, concordância de $90 \%$ para cárie coronária e doença periodontal. Foi observada concordância intraexaminador de $96,5 \%$ a $100 \%$, estando dentro dos padrões de confiabilidade ${ }^{16}$. O índice Kappa variou de 0,89 a 1.

As condições clínicas de saúde bucal pesquisadas foram: cárie dentária coronária, necessidade de tratamento e condição periodonta ${ }^{17}$. A experiência de cárie foi avaliada através do índice CPOD, obtido através da soma de dentes cariados, perdidos e obturados. A presença de biofilme dental foi avaliada segundo Ainamo e Bay ${ }^{18}$, considerando sim para uma ou mais superfícies dentárias com biofilme visível.

Para a nova classificação da perda dentária, foram excluídos os dentes congenitamente ausentes e os extraídos por motivos ortodônticos, sendo considerados os dentes pedidos por doença cárie e doença periodontal. A classificação foi realizada com seis categorias baseadas no número de dentes perdidos e na posição que ocupavam na boca ${ }^{19}$, e levou em consideração a teoria do arco dentário reduzido ${ }^{20}$.

Classificação da perda dentária:

1. Não perdeu nenhum dente por cárie ou doença periodontal,
2. Perdeu de 1 a 4 primeiros molares permanentes,

3. Perdeu até 12 dentes posteriores que não sejam os 4 primeiros molares,

4. Perdeu até 12 dentes incluindo um ou mais anteriores,

5. Perdeu de 13 a 31 dentes

6. Edêntulos.

Cada voluntário respondeu a um questionário para verificação de fatores demográficos, socioeconômicos e de utilização de serviço odontológico. O questionário foi realizado através de entrevista no momento da realização do exame domiciliar. Foi realizado um estudo piloto previamente a coleta de dados para avaliar a compreensão das questões aplicadas.

\section{Análises estatísticas}

Os dados foram tabulados no Statistical Package for the Social Sciences (SPSS), versão 20.0 e no Excel ${ }^{\circledR}$. Realizou-se uma análise descritiva obtendo-se a distribuição absoluta e percentual, média, mediana e desvio padrão (DP) das variáveis, que foram as condições examinadas. Para a distribuição das condições de perdas dentárias, a amostra foi estratificada em dois grupos etários, de 20 a 44 anos e de 45 a 64 anos.

O desfecho do estudo foi a perda dentária, avaliado através da nova classificação proposta. Para a realização das análises de regressão logística nominal houve agrupamento das seis categorias em quatro, sendo: 1- Não perdeu nenhum dente por cárie ou doença periodontal, 2- Perdeu até 12 dentes posteriores (categoria 2 e 3), 3-Perdeu até 12 dentes incluindo anteriores, 4 - Perdeu acima de 12 dentes (categoria 5 e 6). O agrupamento foi realizado porque as categorias: perdeu apenas os primeiros molares e edêntulos, apresentaram um número de indivíduos insuficiente para a realização da análise.

Foi utilizado um modelo teórico conceitual adaptado de "Aging, Ethnicity and Oral Health Outcomes" que foi proposto por Andersen e Davidson $^{21}$ para perdas dentárias adaptado por Batista et al. ${ }^{19}$ (Figura 1). Este modelo foi utilizado para direcionar a análise hierárquica ${ }^{22}$ em quatro blocos. Foram realizadas análises bivariadas entre o desfecho e as variáveis independentes do modelo conceitual teórico. Após o ajuste das variáveis em cada bloco, as variáveis que apresentaram $\mathrm{p}<0,20$ foram selecionadas para ajustar o bloco subsequente, até a obtenção do modelo final. A categoria de referência para a regressão logística multinomial foi não ter perdido dentes por cárie 


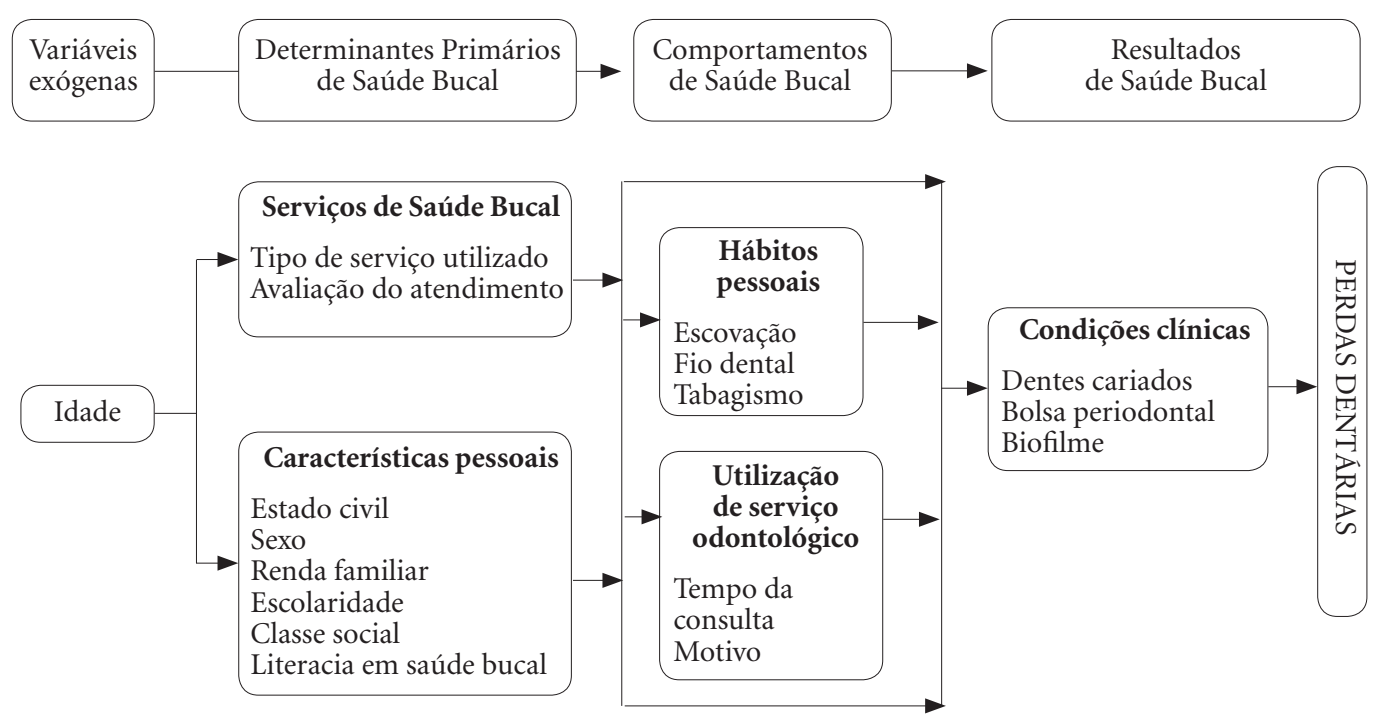

Figura 1. Modelo conceitual teórico para perdas dentárias adaptado para o estudo.

Fonte: Aging, Ethnicity and Oral Health Outcomes, Andersen e Davidson ${ }^{21}$.

ou doença periodontal. Foi adotado o nível de significância de $5 \%$.

No primeiro bloco, a variável idade foi considerada como anos de vida completos. No segundo bloco, onde estão as variáveis determinantes primárias da saúde bucal, o tipo de serviço odontológico foi: público, particular e convênio; a avaliação do serviço utilizado foi classificada como boa ou não boa; o estado civil foi dicotomizado entre os que vivem em união estável, ou não; o sexo em masculino e feminino; a renda em alta [acima de 3 salários mínimos (SM) cujo valor era $\mathrm{R} \$ 622,00$ na época do estudo], média (2-3 SM) ou baixa (até $1 \mathrm{SM}) ;$ A escolaridade foi classificada segundo os anos de estudo, em até 4 anos, de 5 a 11 anos e acima de 11 anos. A classe social foi avaliada segundo a classificação de Graciano et al. ${ }^{23}$, que utiliza uma pontuação baseada na escolaridade, renda familiar, ocupação, tipo de residência e número de residentes no domicílio, agrupando as pontuações em seis classes sociais. Para este estudo, as classes foram agrupadas em média inferior-baixa e média superior-alta. Com relação as informações sobre saúde bucal foi verificado se o indivíduo recebeu ou não informações, bem como foi avaliado se o mesmo tinha literacia em saúde bucal alta ou bai$\mathrm{xa}^{24}$. A literacia em saúde neste estudo foi medida através de 5 questões, cujos resultados foram pon- tuados em escala Lickert, para avaliar a capacidade de julgar e utilizar as informações em saúde bucal recebidas, segundo o questionário proposto por Ishikawa et al. ${ }^{24}$. Após o total obtido através da soma das 5 questões, a literacia foi dicotomizada na mediana em uma variável que avalia a baixa ou alta literacia.

No terceiro bloco Comportamentos em saúde bucal, foram avaliados como práticas pessoais de saúde e utilização de serviço odontológico: escovar os dentes (até duas vezes por dia e 3 ou mais), o uso de fio dental (regular ou não) e fumar (sim ou não). Foi verificada o tempo da última vez que visitou o dentista (menos que 1 ano, 1-2 anos, e 3 ou mais anos), e o motivo da visita, que foi agrupado em dor, rotina, ou outros.

No quarto bloco foram analisados os resultados de saúde bucal, que foram a presença de bolsa periodontal até $4 \mathrm{~mm}$ ou mais, ter ou não lesões cariosas não tratadas e presença de biofilme dental visível (sim ou não).

\section{Resultados}

Foram examinados 248 adultos de 20 a 64 anos. A taxa de resposta dos domicílios foi $75,15 \%$, havendo perda de 82 residências. Entretanto, 
atingiu-se o mínimo necessário calculado para o tamanho amostral de adultos.

A Tabela 1 apresenta as características demográficas e socioeconômicas dos adultos de Piracicaba. A maioria dos examinados foi mulheres, vivendo em união estável. Completaram pelo menos ensino médio $54,8 \%$ dos adultos e $21,8 \%$ apresentaram renda pessoal média.

A média de dentes cariados foi $1,04( \pm 2,06)$, de dentes perdidos foi 8,52 ( $\pm 9,24)$, sendo que para os mais jovens, de 20 a 44 anos, foi 3,30 ( \pm 3,77 ), e para os adultos de 45 a 64 foi 14,68 ( \pm $9,97)$. A média de dentes restaurados foi $8,68( \pm$ $6,29)$. O CPOD para a amostra examinada foi $18,02( \pm 8,89)$ havendo diferença entre adultos mais jovens que apresentou CPOD $=12,94( \pm$ $7,26)$ e mais os velhos CPOD $=24,49( \pm 6,16)$.
Observou-se, através dos dados apresentados, que a diferença na experiência de cárie dos adultos ocorre principalmente devido ao número de dentes perdidos.

$\mathrm{Na}$ Tabela 2 pode se observar características dessa população estudada com relação às perdas dentárias. De acordo com a classificação de perdas dentárias, 28,3\% $(\mathrm{n}=70)$ não perderam dentes devido às doenças bucais, $4,9 \%(\mathrm{n}=12)$ perderam de 1 a 4 primeiros molares, 18,2\% (n = 45) perderam até 12 dentes posteriores, $25,1 \%$ $(\mathrm{n}=62)$ perderam até 12 dentes, incluindo um/ mais anteriores, $18,2 \%(n=45)$ perderam de 13 31 dentes, e 5,3\% $(n=13)$, eram desdentados. $\mathrm{Na}$ Tabela 2 estão os resultados da classificação das perdas dentárias como foi agrupada para as análises de regressão nominal.

Tabela 1. Características demográficas, socioeconômicas e de práticas em saúde dos adultos residentes em Piracicaba, Brasil, 2011.

\begin{tabular}{|c|c|c|c|}
\hline Variáveis & $\mathbf{n} / \%$ & Variáveis & $\mathbf{n} / \%$ \\
\hline Variáveis exogénas & & Comportamentos em saúde bucal & \\
\hline Idade (anos) & & Uso do serviço odontológico & \\
\hline $45-64$ & $110(44,4)$ & Emergência & $132(53,2)$ \\
\hline $20-44$ & $138(55,6)$ & Regular & $113(45,6)$ \\
\hline Determinantes de Saúde Bucal & & Motivo da consulta odontológica & \\
\hline Tipo de serviço utilizado & & Dor & $55(22,2)$ \\
\hline Público & $55(22,2)$ & Necessidade & $54(21,8)$ \\
\hline Convênio & $58(23,4)$ & Rotina & $131(52,8)$ \\
\hline Privado & $131(52,8)$ & Escovação & \\
\hline Avaliação do serviço utilizado & & $\leq 2$ por dia & $89(35,9)$ \\
\hline Não boa & $35(14,1)$ & $3+$ por dia & $159(64,1)$ \\
\hline Boa & $210(84,7)$ & Uso do fio dental & \\
\hline Estado civil & & Irregular & $148(59,7)$ \\
\hline União estável & $174(70,2)$ & Regularmente & $100(40,3)$ \\
\hline Sem união estável & $74(29,8)$ & Fuma & \\
\hline Sexo & & Sim & $48(19,4)$ \\
\hline Feminino & $179(72,2)$ & Não & $200(80,6)$ \\
\hline Masculino & $69(27,8)$ & Condições clínicas & \\
\hline Classe Social & & Dentes cariados & \\
\hline Média Inf- Baixa & $124(50,0)$ & Sim & $91(36,7)$ \\
\hline Média Sup- Alta & $124(50,0)$ & Não & $157(63,3)$ \\
\hline Escolaridade & & Biofilme dental & \\
\hline Até 4 anos & $43(17,3)$ & Sim & $101(40,7)$ \\
\hline 5-11 anos & $69(27,8)$ & Não & $141(56,9)$ \\
\hline+11 anos & $136(54,8)$ & Bolsa Periodontal & \\
\hline Renda pessoal & & $>4 \mathrm{~mm}$ & $82(33,1)$ \\
\hline Baixa & $96(38,6)$ & Até $4 \mathrm{~mm}$ & $166(66,9)$ \\
\hline Média & $54(21,8)$ & & \\
\hline Alta & $94(37,9)$ & & \\
\hline \multicolumn{4}{|l|}{ Literacia em saúde bucal } \\
\hline Baixa & $117(47,2)$ & & \\
\hline Alta & $131(52,8)$ & & \\
\hline
\end{tabular}

Nota: As variáveis que não somam $100 \%$ apresentam dados perdidos que não foram considerados na análise. 
Na Tabela 3, podem ser observados os resultados das análises bivariadas entre a classificação da perda dentária e as variáveis independentes do modelo conceitual teórico. A idade (bloco 1) foi significativa e ajustou escolaridade, classe social e estado civil (bloco 2). Essas quatro variáveis ajustaram o bloco 3, resultando na seleção de idade, frequência da visita ao dentista, classe social e o hábito de fumar, que ajustaram o bloco 4 produzindo o modelo final.

Conforme o aumento da idade a cada ano, a razão da prevalência de perda dentária aumentou de 1,10 a 1,30 , ou seja, de 10 a $30 \%$, nos adultos de Piracicaba. A idade foi associada à perda dentária, à classe social, à frequência de utilização do serviço odontológico e à presença de bolsa periodontal, sendo que houve maior prevalência de utilização do serviço odontológico apenas em emergência entre os que perderam acima de 12 dentes, e houve associação significativa entre ter bolsa periodontal e ter perdido até 12 dentes incluindo anteriores (Tabela 4).

\section{Discussão}

A idade, a classe social, a utilização do serviço odontológico por emergência e a presença de bolsa periodontal foram associados à perda dentária, porém os fatores associados foram diferentes em cada categoria, de acordo com o número de dentes perdidos e posição que os mesmos ocupavam na arcada dentária. De acordo com as categorias da classificação proposta, a utilização do serviço odontológico esteve associada com a perda acima de 12 dentes, e ter bolsa periodontal com a perda de até 12 dentes incluindo anteriores. A utilização de uma classificação que avalie a perda dentária quantitativamente (número de dentes perdidos) e qualitativamente (posição que ocupavam na arcada) na população adulta pode ser bastante útil na compreensão deste fenômeno bem como na identificação dos fatores de risco desta condição que é uma das doenças mais prevalentes do mundo e impacta na qualidade de vida $^{2}$ para o planejamento de estratégias de promoção de saúde.

Um importante conceito para adotar um indicador de saúde, é a clara definição do que é saúde e do que é doença ${ }^{25}$. A classificação da perda dentária proposta neste estudo foi baseada no arco dentário reduzido, que considera satisfatório para o indivíduo ter vinte pares de dentes que ocluem e sem lacunas estéticas ${ }^{20}$. Na literatura, encontram-se estudos que avaliam as perdas dentárias apenas numericamente, utilizando a base de corte na presença de 20 dentes ou mais ${ }^{8,9}$, porém sem considerar a posição dos dentes na arcada dentária, se anterior ou posterior. A medida quantitativa (número de dentes perdidos), entretanto, subestima a posição que os mesmos ocupam na arcada dentária, e esta se mostrou importante para avaliar o impacto da perda dentária na qualidade de vida ${ }^{4}$. O diferencial do presente estudo é avaliar qualitativamente e quantitativamente a perda dentária.

Outro ponto a ser ressaltado nessa nova classificação das perdas dentárias é o fato de considerar as perdas dentárias por cárie e doença periodontal, excluindo os dentes ausentes congenitamente ou por motivos ortodônticos que já era critério da $\mathrm{OMS}^{17}$. A nova classificação da perda dentária pode auxiliar na interpretação da distribuição desta condição em um estudo epidemiológico, que usualmente é mensurada, apenas considerando o componente perdidos $(\mathrm{P}) \mathrm{da}$

Tabela 2. Edentulismo e classificação da perda dentária estratificada por grupo etário em adultos residentes em Piracicaba, SP, Brasil, 2011.

\begin{tabular}{|c|c|c|c|}
\hline Perdas dentárias & $\begin{array}{l}\text { Total } \\
\%(\mathbf{n})\end{array}$ & $\begin{array}{c}20-44 \text { anos } \\
\%(n)\end{array}$ & $\begin{array}{c}\text { 45-64 anos } \\
\%(n)\end{array}$ \\
\hline Média (DP) de dentes presentes & $22,81( \pm 8,91)$ & $27,49( \pm 4,36)$ & $16,94( \pm 9,67)$ \\
\hline \% Edêntulos & $5,6(14)$ & $0,7(1)$ & $11,8(13)$ \\
\hline$\%$ com 32 dentes & $5,6(14)$ & $9,4(13)$ & $0,9(1)$ \\
\hline$\%$ com menos que 21 dentes & $27,4(68)$ & $6,5(9)$ & $53,6(59)$ \\
\hline \multicolumn{4}{|l|}{ Classificação das perdas dentárias } \\
\hline Não apresentou perdas dentárias por doença bucal & $28,3(70)$ & $47,4(65)$ & $4,5(5)$ \\
\hline Perdeu até 12 dentes posteriors & $23,1(57)$ & $27,7(38)$ & $17,3(19)$ \\
\hline Perdeu até 12 dentes incluindo anteriores & $25,1(62)$ & $21,9(30)$ & $29,1(32)$ \\
\hline Perdeu acima de 12 dentes & $23,5(58)$ & $2,9(4)$ & $49,1(54)$ \\
\hline
\end{tabular}


Tabela 3. Análises bivariadas para a classificação da perda dentária, Odds Ratio (OR) bruto, intervalo de confiança 95\% e valor de p, em adultos residentes em Piracicaba, Brasil, 2011.

\begin{tabular}{|c|c|c|c|c|c|c|c|c|c|}
\hline \multirow{2}{*}{$\begin{array}{c}\text { Classificação da perda } \\
\text { dentária }\end{array}$} & \multicolumn{3}{|c|}{ Perdeu até 12 dentes } & \multicolumn{3}{|c|}{$\begin{array}{l}\text { Perdeu até } 12 \text { dentes } \\
\text { incluindo anteriores }\end{array}$} & \multicolumn{3}{|c|}{ Perdeu acima de 12 dentes } \\
\hline & $\begin{array}{c}\text { OR } \\
\text { bruto }\end{array}$ & IC $95 \%$ & $\mathbf{p}$ & $\begin{array}{c}\text { OR } \\
\text { bruto }\end{array}$ & IC $95 \%$ & p & $\begin{array}{c}\text { OR } \\
\text { bruto }\end{array}$ & IC 95\% & $\mathrm{p}$ \\
\hline \multicolumn{10}{|l|}{ Variáveis exogénas } \\
\hline Idade (anos) & 1,09 & $1,05-1,13$ & $<0,001$ & 1,13 & $1,09-1,18$ & $<0,001$ & 1,27 & $1,20-1,35$ & $<0,001$ \\
\hline \multicolumn{10}{|c|}{ Determinantes de Saúde Bucal } \\
\hline \multicolumn{10}{|l|}{ Tipo de serviço utilizado } \\
\hline Público & 1,97 & $0,80-4,87$ & 0,141 & 1,53 & $0,61-3,83$ & 0,362 & 1,50 & $0,62-3,65$ & 0,363 \\
\hline Convênio & 1,68 & $0,71-3,97$ & 0,235 & 1,70 & $0,74-3,91$ & 0,214 & 0,72 & $0,28-1,86$ & 0,501 \\
\hline Privado & 1 & & & 1 & & & 1 & & \\
\hline \multicolumn{10}{|l|}{ Avaliação do serviço } \\
\hline Não boa & 1,33 & $0,48-3,69$ & 0,582 & 0,93 & $0,37-2,38$ & 0,883 & 1,33 & $0,48-3,69$ & 0,582 \\
\hline Boa & 1 & & & 1 & & & 1 & & \\
\hline \multicolumn{10}{|l|}{ Estado civil } \\
\hline União estável & 3,13 & $1,36-7,21$ & 0,007 & 1,51 & $0,73-3,10$ & 0,264 & 1,75 & $0,83-3,70$ & 0,143 \\
\hline Sem união estável & 1 & & & 1 & & & 1 & & \\
\hline \multicolumn{10}{|l|}{ Sexo } \\
\hline Feminino & 1,12 & $0,51-2,46$ & 0,777 & 1,37 & $0,62-3,02$ & 0,433 & 0,760 & $0,36-1,61$ & 0,473 \\
\hline Masculino & 1 & & & 1 & & & 1 & & \\
\hline \multicolumn{10}{|l|}{ Renda pessoal } \\
\hline Baixa & 1,19 & $0,53-2,66$ & 0,670 & 1,48 & $0,67-3,26$ & 0,329 & 1,96 & $0,87-4,38$ & 0,103 \\
\hline Média & 0,77 & $0,30-1,99$ & 0,585 & 1,20 & $0,50-2,92$ & 0,682 & 1,18 & $0,46-3,02$ & 0,735 \\
\hline Alta & 1 & & & 1 & & & 1 & & \\
\hline \multicolumn{10}{|l|}{ Escolaridade } \\
\hline Até 4 anos & 2,30 & $0,49-10,99$ & 0,294 & 8,58 & $2,29-32,17$ & 0,001 & 29,86 & $7,81-114,10$ & $<0,001$ \\
\hline 5-11 anos & 3,46 & $1,45-8,26$ & 0,005 & 3,13 & $1,28-7,65$ & 0,013 & 8,96 & $3,47-23,14$ & $<0,001$ \\
\hline+11 anos & 1 & & & 1 & & & 1 & & \\
\hline \multicolumn{10}{|l|}{ Classe social } \\
\hline Média Inf- Baixa & 2,44 & $1,18-5,01$ & 0,016 & 1,92 & $0,95-3,88$ & 0,071 & 4,54 & $2,15-9,59$ & $<0,001$ \\
\hline Média Sup- Alta & 1 & & & 1 & & & 1 & & \\
\hline \multicolumn{10}{|l|}{ Literacia } \\
\hline Baixa & 0,77 & $0,37-1,59$ & 0,482 & 0,49 & $0,24-0,99$ & 0,046 & 0,275 & $0,13-0,57$ & 0,001 \\
\hline Alta & 1 & & & 1 & & & 1 & & \\
\hline
\end{tabular}

experiência de cárie (índice CPOD) (ver Tabela 2). Clinicamente, com a incorporação de dentes perdidos por doença periodontal, esta nova classificação permite identificar a questão estética e funcional, importantes também para o planejamento de reabilitação oral dos pacientes.

A presença de bolsa periodontal acima de $4 \mathrm{~mm}$ em um ou mais sextantes foi associada à perda de até 12 dentes incluindo um ou mais dentes anteriores e segundo Petersen ${ }^{26}$ a doença periodontal mais severa pode estar relacionada com 5 a $15 \%$ das perdas dentárias na maioria dos estudos epidemiológicos. Fato que pode ser ocultado utilizando indicadores baseados apenas na experiência de cárie para avaliar a perda dentária. 


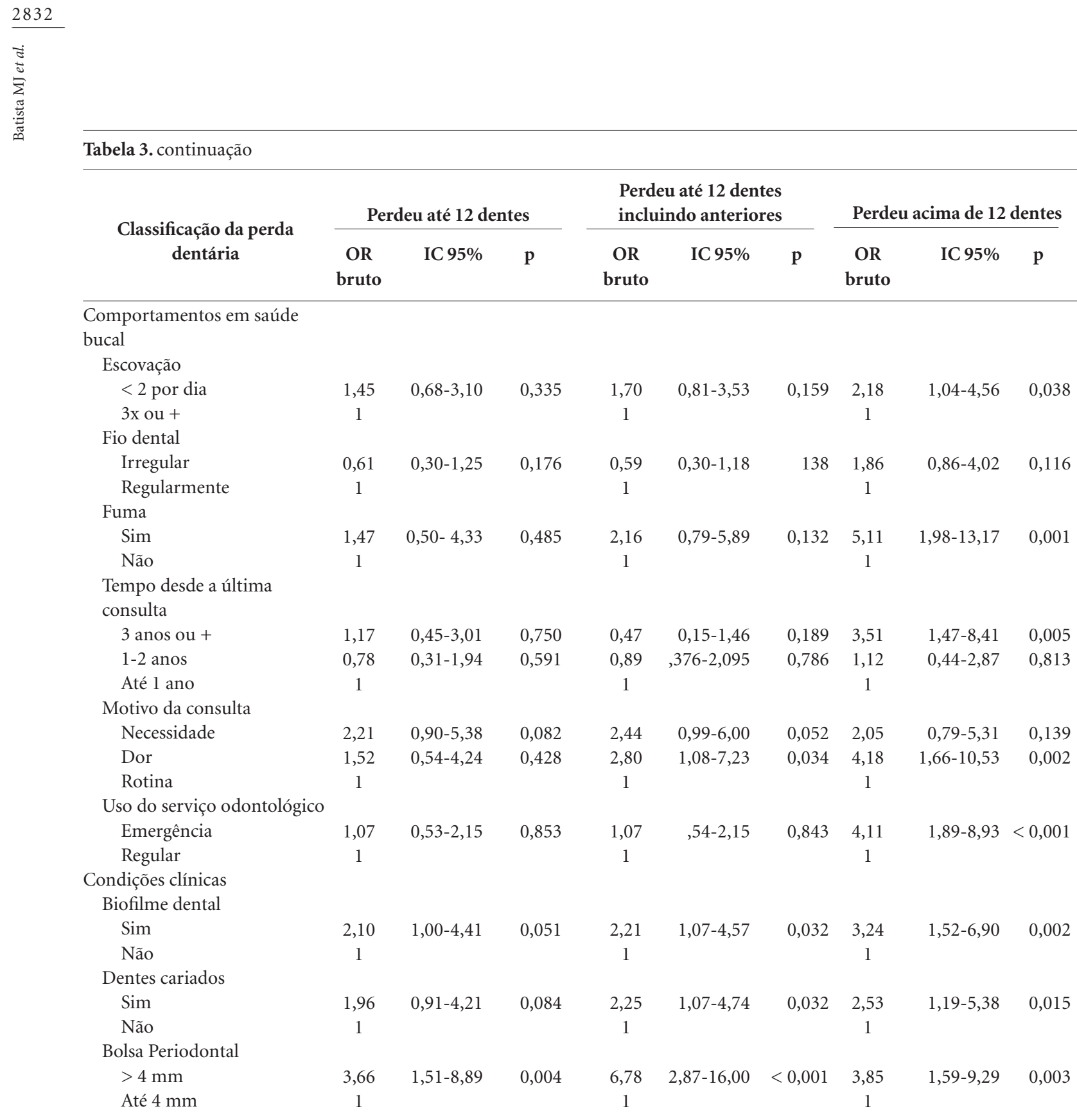

Nota: A categoria de referência para a análise de regressão multinomial foi "Não ter perdido nenhum dente por cárie ou doença periodontal”.

de bucal na Estratégia de Saúde da Família que ocorreu em 2000, e também com a criação dos Centros de Especialidades Odontológicas, em 2004, que proporcionam serviços de endodontia, periodontia e prótese ${ }^{28}$. Em Piracicaba, mesmo que esta estratégia governamental tenha sido implantada mais recentemente, os adultos podem se beneficiar da ampliação do acesso à atenção secundária, que possibilita tratamentos odontológicos mais especializados evitando assim, a perda do dente em uma extração. Nos resultados do último levantamento de saúde bucal realizado no Brasil, a população adulta apresentou índice mais baixo de experiência de cárie e, assim, redução nos dentes perdidos ${ }^{6}$, em relação aos levantamentos epidemiológicos anteriores ${ }^{5,29}$, embora a prevalência ainda seja alta.

Além da idade, a prevalência das perdas dentárias mais elevadas esteve associada à condição socioeconômica. A utilização da classe social segundo Graciano et al. ${ }^{23}$, composta por renda familiar, escolaridade, ocupação, tipo de residência e número de residentes no domicílio, teve como objetivo avaliar o nível socioeconômico através 
Tabela 4. Indicadores de risco da perda dentária utilizando uma nova classificação em adultos de 20 a 64 anos, Piracicaba, Brasil, 2011.

\begin{tabular}{|c|c|c|c|}
\hline Classificação da perda dentária & OR ajustado & IC $95 \%$ & $\mathbf{p}$ \\
\hline \multicolumn{4}{|l|}{ Perdeu até 12 dentes posteriores } \\
\hline \multicolumn{4}{|l|}{ Idade } \\
\hline Cada ano & 1,10 & $1,04-1,13$ & $<0,001$ \\
\hline Classe social & 2,62 & $1,17-5,16$ & 0,019 \\
\hline \multicolumn{4}{|l|}{ Média Inf- Baixa } \\
\hline \multicolumn{4}{|l|}{ Média Sup- Alta } \\
\hline \multicolumn{4}{|l|}{ Uso do serviço odontológico } \\
\hline Emergência & 1,14 & $0,51-2,53$ & 0,748 \\
\hline \multicolumn{4}{|l|}{ Regularmente } \\
\hline \multicolumn{4}{|l|}{ Bolsa periodontal } \\
\hline$>4 \mathrm{~mm}$ & 1,99 & $0,76-5,18$ & 0,160 \\
\hline \multicolumn{4}{|l|}{$<4 \mathrm{~mm}$} \\
\hline \multicolumn{4}{|l|}{ Perdeu até 12 dentes incluindo anteriores } \\
\hline \multicolumn{4}{|l|}{ Idade } \\
\hline Cada ano & 1,13 & $1,08-1,19$ & $<0,001$ \\
\hline \multicolumn{4}{|l|}{ Classe social } \\
\hline Média Inf- Baixa & 2,08 & $0,88-4,93$ & 0,095 \\
\hline \multicolumn{4}{|l|}{ Média Sup- Alta } \\
\hline \multicolumn{4}{|l|}{ Uso do serviço odontológico } \\
\hline Emergência & 1,47 & $0,63-3,45$ & 0,377 \\
\hline \multicolumn{4}{|l|}{ Regularmente } \\
\hline \multicolumn{4}{|l|}{ Bolsa periodontal } \\
\hline$>4 \mathrm{~mm}$ & 2,86 & $1,08-7,52$ & 0,034 \\
\hline \multicolumn{4}{|l|}{$<4 \mathrm{~mm}$} \\
\hline \multicolumn{4}{|l|}{ Perdeu acima de 12 dentes } \\
\hline \multicolumn{4}{|l|}{ Idade } \\
\hline Cada ano & 1,30 & $1,22-1,39$ & $<0,001$ \\
\hline \multicolumn{4}{|l|}{ Classe social } \\
\hline Média Inf- Baixa & 3,84 & $1,33-11,44$ & 0,013 \\
\hline \multicolumn{4}{|l|}{ Média Sup- Alta } \\
\hline \multicolumn{4}{|l|}{ Uso do serviço odontológico } \\
\hline Emergência & 9,37 & $3,03-28,95$ & $<0,001$ \\
\hline \multicolumn{4}{|l|}{ Regularmente } \\
\hline \multicolumn{4}{|l|}{ Bolsa periodontal } \\
\hline$>4 \mathrm{~mm}$ & 1,22 & $0,39-3,86$ & 0,730 \\
\hline$<4 \mathrm{~mm}$ & & & \\
\hline
\end{tabular}

Nota: A categoria de referência para a análise de regressão multinomial foi "Não ter perdido nenhum dente por cárie ou doença periodontal".

de uma única variável, evitando a colinearidade com as demais nas análises de regressão. No presente estudo, os adultos que pertenciam às classe sociais mais baixas apresentaram mais perdas dentárias, independente da posição do dente perdido na arcada dentária. Estes dados corroboram com outros estudos que encontraram associação entre perdas dentárias e fatores socioeconômicos no Brasil $1^{8,9,11,12,27,30}$. A influência da questão socioeconômica na saúde bucal reforça a ocorrência das iniquidades em saúde. Sendo que os grupos que encontram-se em desvantagem socialmente e economicamente, apresentam também as piores condições de saúde e o maior impacto das doenças bucais na qualidade de vida ${ }^{31,32}$. Watt e Sheiham ${ }^{33}$ observaram que a condição de saúde bucal é diretamente relacionado com os gradientes da posição socioeconômica na sociedade, sendo que a prevalência de edentulismo aumenta conforme os gradientes das classes de ocupação diminuem. Além disso, as questões socioeconômicas estão também relacionadas aos comportamentos e práticas em saúde, como, por exemplo, a utilização de serviço odontológico. 
A utilização de serviço apenas por emergência foi associada à perda acima de 12 dentes. Do ponto de vista biomédico, a questão da utilização de serviço por emergência, revela a procura por cuidado quando a doença bucal já está instalada e causando dor ou incômodo, que revelam estar em estágio já avançado ${ }^{3}$. Dessa maneira, as opções de tratamento em alternativa a exodontia, quando existem, são onerosas, o que acaba culminando com a perda dental. Silva et al. ${ }^{9}$ encontrou associação entre procurar o dentista motivado pela dor e ter maior prevalência de perdas dentárias. Porém, por trás do comportamento e do modelo biomédico, a utilização irregular dos serviços de saúde está fortemente relacionada à desvantagem social.

A Organização Mundial de Saúde tem enfatizado a importância do desenvolvimento de políticas públicas para reduzir as vulnerabilidades das populações em desvantagem, que continuam sofrendo com doenças bucais, que poderiam ser evitadas e prevenidas ${ }^{32}$. Considerar a abordagem populacional em detrimento da abordagem individual centrada no diagnóstico e tratamento restaurador e reabilitador pode ser uma importante estratégia para a melhoria da saúde bucal ${ }^{13}$.

Este estudo transversal não tem o poder de realizar inferência causal. É um estudo que conta com uma amostra representativa obtida através de uma amostragem probabilística, e aborda uma ampla faixa etária de adultos, que não está incluída na preconização da OMS para levantamentos epidemiológicos, embora apresente uma maior porcentagem de mulheres, que pode ser explicada pelo fato de ser um estudo domiciliar. Uma limitação encontrada foi à distribuição desta população entre as categorias da perda dentária. Para possibilitar as análises estatísticas, houve necessidade de agrupar a classificação da perda dentária em quatro categorias. São necessários estudos futuros em diferentes populações, tendo em vista que o município foi realizado em apenas um município, para avaliar como se dá essa distribuição, considerando a perda dentária em aspectos qualitativos e quantitativos.

\section{Conclusão}

As análises mostraram que idade e classe social baixa foram fatores associados à perda dentária. As categorias da nova classificação da perda dentária, que considera a posição e o número de dentes perdidos, permitiram identificar condições clínicas e fatores de comportamento, como a utilização de serviço odontológico. A avaliação da perda dentária pode ser subestimada se não for quantitativa e qualitativa ao mesmo tempo, devendo ser considerada em futuros estudos.

\section{Colaboradores}

MJ Batista, HP Lawrence e MLR Sousa participaram igualmente de todas as fases do artigo. 


\section{Referências}

1. Elderton RJ. Preventive (Evidence-Based) approach to quality general dental care. Med Princ Pract 2003; 12(Supl. 1):12-21

2. Marcenes W, Kasseabaum NJ, Barnabé E, Flaxman A, Naghavi M, Lopez A, Murray CJL. Global Burden of oral conditions in 1990-2010: A Systematic Analysis. J Dent Res 2013; 92(7):592-597.

3. Gerritsen AE, Allen PF, Witter DJ, Bronkhorst EM, Creugers NHJ. Tooth loss and oral health-related quality of life: a systematic review and meta-analysis. Health and Quality of Life Outcomes 2010; 8:126.

4. Batista M, Lawrence H, Sousa MLR. Impact of tooth loss related to number and position on oral health quality of life among adults. Health Qual Life Outcomes 2014; 12(1):165.

5. Brasil. Ministério da Saúde (MS). Secretaria Nacional de Programas Especiais de Saúde. Divisão Nacional de Saúde Bucal. Fundação Serviços de Saúde Pública. Levantamento Epidemiológico em Saúde Bucal: Brasil, Zona Urbana, 1986. Brasília: MS; 1988. (Série C: estudos e projetos, 4).

6. Brasil. Ministério da Saúde (MS). Secretaria de Atenção à Saúde. Departamento de Atenção Básica. Projeto SB Brasil 2010: condições de saúde bucal da população brasileira 2010-2011: resultados principais. Brasília: MS; 2011. (Série C. Projetos, Programas e Relatórios).

7. Fure S. Ten-year incidence of tooth loss and dental caries in elderly Swedish individuals. Caries Res 2003; 37(6):462-469.

8. Barbato PR, Nagano HCM, Zanchet FN, Boing AF, Peres MA. Tooth loss and associated socioeconomic, demographic, and dental-care factors in Brazilian adults: an analysis of the Brazilian Oral Health Survey, 20022003. Cad Saude Publica 2007; 23(8):1803-1814.

9. Silva DD, Rihs LB, Sousa MRL. Factors associated of maintenance of teeth in adults in the state of São Paulo, Brazil. Cad Saude Publica 2009; 25(11):2407-2418.

10. Corraini P, Baelum V, Pannuti CM, Pustiglioni AN, Romito GA, Pustiglioni FE. Tooth loss prevalence and risk indicators in an isolated population of Brazil. Acta Odontol Scand 2009; 67(5):297-303.

11. Moreira RS, Nico LS, Barrozo LV, Pereira JCR. Tooth Loss in Brazilian middle-aged adults: multilevel effects. Acta Odontol Scand 2010; 68(5):269-277.

12. Batista MJ, Rihs LB, Sousa MLR. Risk indicators for tooth loss in adult workers. Braz O Research 2012; 26(5):390-396.

13. McGrath C, Lawrence HP, Blinkhorn A. Guest editorial on the Festchrift "Challenges in population oral health for the $21^{\text {st }}$ Century". Community Dent Oral Epidemiol 2012; 40(Supl. 2):1-4.

14. Batchelor P. What do we mean by population health? Community Dent Oral Epidemiol 2012; 40(Supl. 2):1215.

15. Instituto Brasileiro de Geografia e Estatísticas (IBGE) [acessado 2010 mar 1]. Disponível em: http://www. ibge.gov.br.

16. Eklund SA, Moller IJ, Leclercq MH. Calibrating examiners for oral health epidemiological surveys. Geneva: World Health Organization (WHO/ORH/Epid.93.1); 1996.

17. World Health Organization (WHO). Basic methods. Geneva: WHO; 1987.
18. Ainamo J, Bay I. Problems and proposals for recording gingivitis and plaque. Int Dent J 1975; 25(4):229-235.

19. Batista MJ. Perda dentária em adultos: avaliação do impacto na qualidade de vida através da aplicação de índices de perda dentária [tese]. Campinas: Universidade Estadual de Campinas; 2013.

20. Armellini D, von Fraunhofer JA. The shortened dental arch: A review of the literature. J Prosthetic Dentistry 2002; 92(6):531-535.

21. Andersen RM, Davidson PL. Ethnicity, aging, and oral health outcomes: A conceptual framework. Adv Dent Res 1997; 11(2):203-209.

22. Victora CG, Huttly SR, Fuchs SC, Olinto MTA. The role of conceptual frameworks in epidemiological analysis: a hierarchical approach. Int J Epidemiol 1997; 26(1):224-227.

23. Graciano MIG, Lehfeld NA, Neves Filho A. Critérios de avaliação para classificação socioeconômica: elementos de atualização. Serviço Social e Realidade 1999; 8(1):109-128.

24. Ishikawa H, Nomura K, Sato M, Yano E. Developing a measure of communicative and critical health literacy: a pilot study of Japanese office workers. Health Promot Int 2008; 23(3):269-274.

25. Mejia GC. Measuring the oral health of populations. Community Dent Oral Epidemiol 2012; 40(Supl. 2):95101.

26. Petersen PE. The World Oral Health Report 2003: continuous improvement of oral health in the $21^{\text {st }}$ century: the approach of the WHO Global Oral Health Programme. Community Dent Oral Epidemiol 2003; 31(Supl. 1):3-23.

27. Borges CM, Campos AC, Vargas AM, Ferreira EF Adult tooth loss profile in accordance with social capital and demographic and socioeconomic characteristics. Cien Saude Colet 2014; 19(6):1849-1858.

28. Antunes JLF, Narvai PC. Políticas de saúde bucal no Brasil e seu impacto sobre as desilgualdades em saúde. Dental health policies in Brazil and their impacto on health inequalities. Rev Saude Publica 2010; 44(2):360365.

29. Brasil. Ministério da Saúde. [SB Brasil 2003: oral health conditions $\mathrm{n}$ the Brazilian population 2002/2003: main results.] Brasília: Ministry of Health; 2004. 68 p. [cited 2009 Oct 15]. Available from: http://dab.saude.gov.br/ cnsb/vigilancia.php

30. Cimões R, Caldas Júnior AF, Souza EHA, Gusmão ES. Influence of social class on clinical reasons for tooth loss. Cien Saude Colet 2007; 12(6):1691-1696.

31. Marmot M. Social Determinants of health inequalities. Lancet 2005; 365(9464):1099-104.

32. Watt RG. Social determinants of oral health inequalities: implications for action. Community Dent Oral Epidemiol 2012; 40(Supl. 2):44-48.

33. Watt RG, Sheiham A. Integrating the common risk factor approach into a social determinants framework. Community Dent Oral Epidemiol 2012; 40(4):289-296.

Artigo apresentado em 09/05/2014

Aprovado em 08/12/2014

Versão final apresentada em 10/12/2014 